\title{
GLUT4, AMP kinase, but not the insulin receptor, are required for hepatoportal glucose sensor-stimulated muscle glucose utilization
}

\author{
Rémy Burcelin, ${ }^{1,2}$ Valerie Crivelli, ${ }^{1}$ Christophe Perrin, ${ }^{2}$ Anabela Da Costa, ${ }^{1}$ James Mu, ${ }^{3}$ \\ Barbara B. Kahn, ${ }^{4}$ Morris J. Birnbaum, ${ }^{3}$ C. Ronald Kahn, ${ }^{5}$ Peter Vollenweider, ${ }^{6}$ \\ and Bernard Thorens ${ }^{1}$
}

\begin{abstract}
${ }^{1}$ Institute of Pharmacology and Toxicology, University of Lausanne, Lausanne, Switzerland
${ }^{2}$ Unite Mixte de Recherche 5018, Rangueil Hospital, Toulouse, France

${ }^{3}$ Department of Medicine and Howard Hughes Medical Institution, University of Pennsylvania, Philadelphia, Pennsylvania, USA

${ }^{4}$ Division of Endocrinology and Metabolism, Beth Israel Deaconess Medical Center and Harvard Medical School, Boston, Massachusetts, USA

${ }^{5}$ Research Division, Joslin Diabetes Center, Department of Medicine, Harvard Medical School, Boston, Massachusetts, USA ${ }^{6}$ Institute of Cell Biology and Morphology, University of Lausanne, Lausanne, Switzerland
\end{abstract}

Recent evidence suggests the existence of a hepatoportal vein glucose sensor, whose activation leads to enhanced glucose use in skeletal muscle, heart, and brown adipose tissue. The mechanism leading to this increase in whole body glucose clearance is not known, but previous data suggest that it is insulin independent. Here, we sought to further determine the portal sensor signaling pathway by selectively evaluating its dependence on muscle GLUT4, insulin receptor, and the evolutionarily conserved sensor of metabolic stress, AMP-activated protein kinase (AMPK). We demonstrate that the increase in muscle glucose use was suppressed in mice lacking the expression of GLUT4 in the organ muscle. In contrast, glucose use was stimulated normally in mice with muscle-specific inactivation of the insulin receptor gene, confirming independence from insulin-signaling pathways. Most importantly, the muscle glucose use in response to activation of the hepatoportal vein glucose sensor was completely dependent on the activity of AMPK, because enhanced hexose disposal was prevented by expression of a dominant negative AMPK in muscle. These data demonstrate that the portal sensor induces glucose use and development of hypoglycemia independently of insulin action, but by a mechanism that requires activation of the AMPK and the presence of GLUT4.

J. Clin. Invest. 111:1555-1562 (2003). doi:10.1172/JCI200316888.

\section{Introduction}

One of the more surprising aspects of normal physiology appreciated only in recent years is that sensors for critical circulating substances can be located in rather surprising anatomical sites and that these can communicate to target organs in nonclassic ways. For example, glucose homeostasis requires that sensing units constantly monitor blood glucose concentrations and modulate food intake and glucose

Received for publication September 11, 2002, and accepted in revised form March 11, 2003.

Address correspondence to: Bernard Thorens, Institute of Pharmacology and Toxicology, University of Lausanne, Rue du Bugnon 27, CH-1005 Lausanne, Switzerland. Phone: 41-21-692-53-90; Fax: 41-21-692-53-55;

E-mail: Bernard.Thorens@ipharm.unil.ch.

Conflict of interest: The authors have declared that no conflict of interest exists.

Nonstandard abbreviations used: 5 '-AMP-activated kinase (AMPK); MGLUT4 (muscle-only GLUT4 gene inactivation); MIRKO (muscle-specific insulin receptor gene inactivation); AMPK-DN (muscle-specific transgenic expression of a dominant negative form of AMPK); ${ }^{14} \mathrm{C}$-2-deoxyglucose $\left({ }^{14} \mathrm{C}-2 \mathrm{DG}\right)$; area under the curve (AUC). use or production. For many years it has been recognized that pancreatic $\beta$ cells possess a well-characterized glucose sensor, which is activated by rises in blood glucose and which induces the secretion of insulin, the hormone most important to the determination of glucose use. Similarly, secretion of glucagon to accelerate hepatic glucose production is triggered by a sensor, which responds to hypoglycemia, present in pancreatic $\alpha$ cells. This secretory activity is also under the control of the autonomic nervous system. Discrete glucose sensors located in the hypothalamus and the brain stem modulate the activity of the autonomic nervous system and regulate feeding and glucose homeostasis.

More recently, the hepatoportal vein has been shown to contain a metabolic sensor that is activated when a glucose gradient is established between the portal vein and the hepatic artery. This sensor stimulates hepatic glucose uptake (1-3), inhibits food intake $(4,5)$, and blocks the secretion of counterregulatory hormones $(6,7)$. These actions are thought to cooperate to minimize postprandial glycemic excursions and regulate termination of food intake. 
Despite clear evidence of the multiple roles of this sensor, numerous questions are still unanswered, including its cellular composition, the way it transmits its signal to target tissues, and the signaling pathways activated at the target tissue level.

In our previous studies (8), we demonstrated that activation of the hepatoportal sensor by a portal glucose infusion led to development of hypoglycemia. This was due to a stimulation of glucose clearance, which was most evident in heart, soleus, and brown adipose tissue. Some information is available concerning the pathway involved in glucose sensing in the hepatoportal circulation. For example, activation of this sensor requires the presence of the glucose transporter isoform GLUT2 (9). Indeed, hypoglycemia and increased glucose clearance could no longer be triggered by portal vein glucose infusion in mice with a $\beta$ cell-specific transgenic expression of GLUT1 to restore a normal insulin secretory function, but with an inactive endogenous GLUT2 gene (10). Furthermore, a functional GLP-1 receptor is required for the glucose competence of the sensor. Indeed, activation by portal glucose infusion is blocked by coinfusion of the GLP-1 antagonist exendin-(9-39) and is undetectable in GLP-1 receptor knockout mice (11). In marked contrast, essentially nothing is known concerning the mechanism by which the hepatoportal sensor activates glucose clearance. It is likely not due to enhanced stimulation of insulin secretion, however, since plasma insulin levels were similar when glucose was infused into the portal or the femoral veins (8), though the latter route of glucose administration was associated with a transient hyperglycemia instead of development of hypoglycemia. It is still possible, however, that sensitization to the action of insulin explains the increase in glucose use or that the process is insulin-independent.

The increase in glucose uptake by muscle in response to insulin or contraction depends on the translocation of intracellular GLUT4 containing vesicles to, and fusion with, the plasma membrane $(12-15)$. Following contraction or hypoxia, the increase in hexose uptake into muscle appears to rely on the activation of $5^{\prime}$-AMP-activated kinase (AMPK). The role of this kinase on the translocation of GLUT4 to the plasma membrane has been demonstrated using AICAR, an activator of AMP kinase, which induces surface expression of the transporter and increases the rate of glucose uptake (16-19). In vivo administration of this substance also induces hypoglycemia $(16,18)$. More recently, mice expressing a transgene encoding a dominant negative form of the $\alpha 2$ subunit of AMPK specifically in muscle displayed decreased GLUT4 translocation and glucose uptake following contraction (20).

Finally, it has been reported that insulin could still stimulate glucose uptake in the soleus of GLUT4-/mice (21). This suggests that other transporter isoforms may be implicated in this insulin-stimulated response, although such increase in glucose uptake was not confirmed in mice with muscle-specific inactivation of GLUT4 (22). Nevertheless, the recent identification of novel members of the glucose transporter family and their localization in muscles $(23,24)$ suggests that GLUT4-independent activation of muscle glucose uptake could exist.

The aim of the present study was to determine, using mice with muscle-specific gene modifications, the mechanisms by which the hepatoportal sensor signal activates glucose uptake in muscle. We determined that stimulated uptake was dependent on the expression of GLUT4 and was independent of the presence of an insulin receptor, but required activation of AMP-activated kinase.

\section{Methods}

Mice and surgical procedures for catheter implantation. The MGLUT4 KO (muscle-only GLUT4 gene inactivation) (22), MIRKO (muscle-specific insulin receptor gene inactivation) (25), and AMPK-DN (muscle-specific transgenic expression of a dominant negative form of AMP kinase) (20) mice were in SV129×C57Bl/6J×FVB, SV129 $\times \mathrm{CD} 1 \times \mathrm{FVB}$, and C57Bl/6J background, respectively. The control mice used in each experiment were the littermates of the respective mutant mice. Under pentobarbital anesthesia $(65 \mathrm{mg} / \mathrm{kg}$ intraperitoneally) a first catheter was implanted into the hepatoportal vein through the splenic vein of 12- to 16 -week-old female control and mutant mice, as described (11). After 4-6 days, allowing body weight recovery, one extremity of a second catheter was implanted into the femoral vein under similar anesthesia. The other extremity of the catheter was tunneled under the skin of the back, exteriorized, and secured in place at the back of the neck. The mice were then allowed to recover from the surgery in individual cages.

Infusion procedures. Five to 7 days after catheter implantation the mice were fasted for $6 \mathrm{~h}$. Saline or glucose was infused for 180 min either through the portal vein, at a rate of approximately $25 \mathrm{mg} / \mathrm{kg} / \mathrm{min}(2 \mu \mathrm{l} /$ $\mathrm{min})$, which corresponds to the mean value of the endogenous glucose production rate in $6-\mathrm{h}$ fasted $30-\mathrm{g}$ mice, as described $(8,9,11)$.

To determine the whole body glucose clearance rate HPLC-purified D- $\left({ }^{3} \mathrm{H}\right) 3$-glucose (NEN Life Science Products Inc., Boston, Massachusetts, USA) was infused continuously through the femoral vein at a rate of $10 \mu \mathrm{Ci} / \mathrm{kg} / \mathrm{min}$. Plasma glucose and D- $\left({ }^{3} \mathrm{H}\right) 3$-glucose concentrations were determined in $5 \mu$ l of blood sampled from the tip of the tail vein each $20 \mathrm{~min}$ following the initiation of infusion. These methods have been validated previously $(8,9,11)$.

Glucose use index in individual tissues. To determine an index for the individual tissue glucose use rate, a flash injection of $1 \mu \mathrm{Ci}$ per mouse of ${ }^{14} \mathrm{C}$-2-deoxyglucose $\left({ }^{14} \mathrm{C}-2 \mathrm{DG}\right.$; NEN Life Science Products Inc.) through the femoral vein was performed $60 \mathrm{~min}$ before the end of the infusions. Plasma ${ }^{14} \mathrm{C}-2 \mathrm{DG}$ disappearance was 
determined in 5- $\mu \mathrm{l}$ drops of blood sampled from the tip of the tail vein at $0,5,10,15,20,25,30,45$, and 60 min after the injection.

Analytical procedures. Blood glucose concentration was determined during the infusions using a glucose meter (Roche Diagnostics A/G, Basel, Switzerland). Plasma insulin was determined using an ELISA kit (Mercodia, Uppsala, Sweden) before and at 10, 30, 50, 120,180 min following the beginning of the infusions in $5 \mu$ l of plasma from $15 \mu$ l of blood collected from the tail tip (8).

Isotope measurements. Plasma D- $\left({ }^{3} \mathrm{H}-3\right)$ glucose enrichment was determined from total blood after deproteinization (8) using $\mathrm{ZnSO}_{4}$ and $\mathrm{Ba}(\mathrm{OH})_{2}$ solutions to precipitate the proteins and blood cells. The $\mathrm{Zn}(\mathrm{OH})_{2}$ precipitate formed was spun down. An aliquot of the supernatant was evaporated to dryness to determine the radioactivity corresponding to $\mathrm{D}-\left({ }^{3} \mathrm{H}-3\right)$ glucose. In another aliquot of the same supernatant glucose concentration was assessed by the glucose oxidase method (Trinder; Sigma-Aldrich, St. Louis, Missouri, USA). Tissue ${ }^{14} \mathrm{C}-2 \mathrm{DG}$ and ${ }^{14} \mathrm{C}-2 \mathrm{DG}-6$ phosphate content were determined as described previously (8). Briefly, a piece of each tissue was dissolved in $1 \mathrm{M}$ $\mathrm{NaOH}$ at $55^{\circ} \mathrm{C}$ for $60-120 \mathrm{~min}$, then neutralized with $1 \mathrm{M}$ hydrochloric acid. ${ }^{14} \mathrm{C}-2 \mathrm{DG}-6$-phosphate and ${ }^{14} \mathrm{C}-2 \mathrm{DG}$ were differentially precipitated by the use of a zinc hydroxide $(0.3 \mathrm{M})$ precipitation or a perchloric acid solution (6\%) (8).

Calculations. Calculations were made with parameters collected during the last $60 \mathrm{~min}$ of the infusions when a steady state $\mathrm{D}-\left({ }^{3} \mathrm{H}\right) 3$-glucose enrichment was obtained. First, glucose turnover was calculated by dividing the $\mathrm{D}-\left({ }^{3} \mathrm{H}\right) 3$-glucose infusion rate by the plasma glucose-specific activity as described (8). The mean was calculated for each time point of this period.
Second, whole body glucose clearance $(\mathrm{ml} / \mathrm{kg} / \mathrm{min})$ was calculated by dividing the mean glucose turnover rate by the mean blood glucose concentration obtained during the last hour of the infusion. The mean value of mice from the same group was reported. The area under the curve (AUC) was calculated for blood glucose and plasma insulin concentrations obtained during the infusion period. To calculate the blood glucose AUC of each mouse, the fasting value was subtracted from each concentration of glucose determined every $20 \mathrm{~min}(t)$ after the beginning of the infusion and every 20 min until completion. The mean values between $t$ and at $t+20$ min were calculated, and the new values were multiplied by $20 \mathrm{~min}$, which corresponds to the time period between two samplings. To calculate the area under the plasma insulin curve the fasted insulinemia was subtracted from each value determined at 10, 30, 50,120, and 180 minutes. The mean values between $t$ and at the corresponding next time period were calculated, and the new values were multiplied by the time length (minutes) between two samplings. These data were then added together to obtain the glucose and insulin areas under the curve.

Statistical analysis. Results are presented as mean plus or minus SE. Statistical significance of differences was analyzed using Student $t$ test for unpaired bilaterally distributed values of unequal variance. Values were considered different from each other when $P$ values were less than 0.05 .

\section{Results}

Time course of blood glucose levels during hepatoportal glucose infusions. To evaluate the mechanism by which the hepatoportal sensor activates glucose use in muscle, we studied mice with muscle-specific deletion of GLUT4 (MGLUT4 KO mice), the insulin
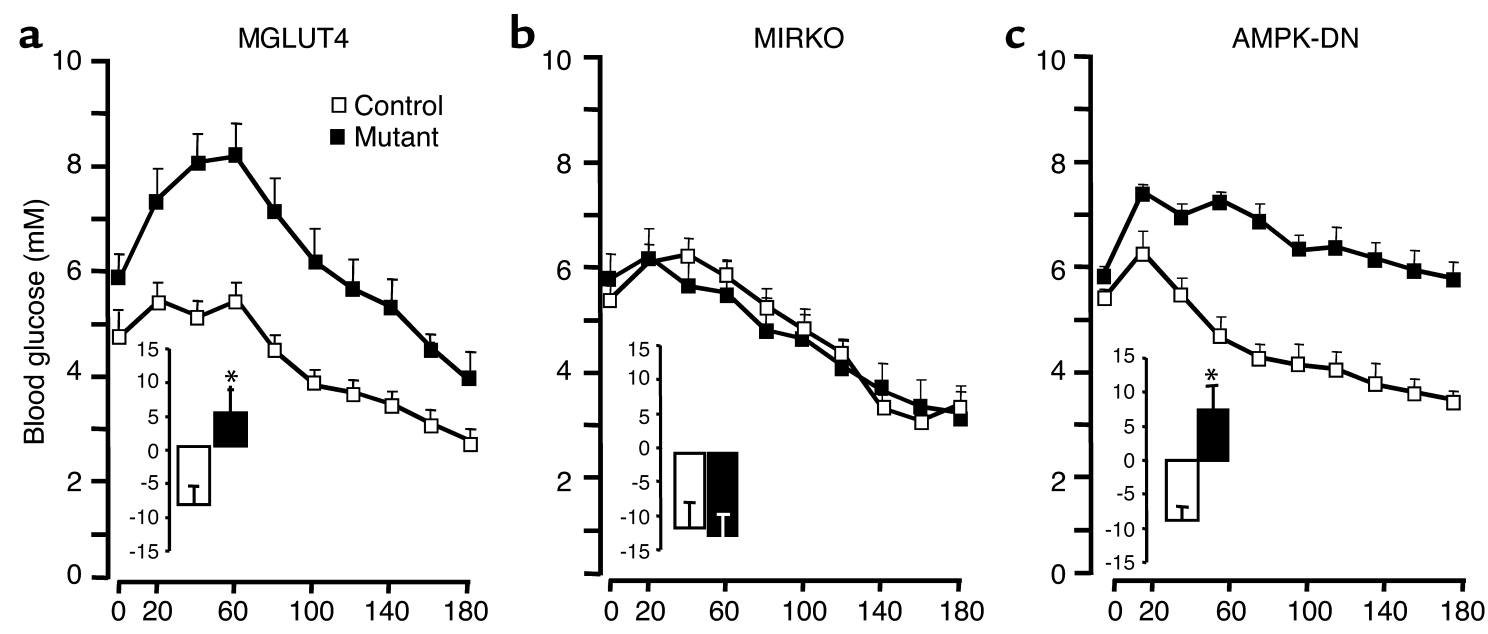

Figure 1

Glycemic profiles of MGLUT4, MIRKO, and AMPK-DN mice and their respective controls infused with glucose into the portal vein. Glucose was infused through the portal vein in control (open squares) and mutant mice (filled squares) for $180 \mathrm{~min}$ at a rate of $\sim 25 \mathrm{mg} / \mathrm{kg} / \mathrm{min}$. The means \pm SE of blood glucose was measured every $20 \mathrm{~min}$. The insets depict the corresponding AUC (mM) for the different groups of mice. (a) MGLUT4 mice; (b) MIRKO mice; (c) AMPK-DN mice. Eight to ten mice were studied per group. ${ }^{*}$ Statistically different from controls; $P<0.05$. 
receptor (MIRKO mice), or with transgenic overexpression of a dominant negative form of the AMP kinase (AMPK-DN mice) and their respective controls. These mice were infused with glucose into the hepatoportal vein and the glycemic profiles were followed over a period of $3 \mathrm{~h}$. As reported previously, hypoglycemia developed progressively in the three different control lines (Figure 1 and Table 1). The extent of hypoglycemia reached at completion of the glucose-infusion periods differed somewhat between the different control mice $(2.8 \pm 0.2,3.3 \pm 0.1$, and 3.4 $\pm 0.2 \mathrm{mM}$ in MGLUT4 KO, MIRKO, AMPK-DN control mice, respectively) probably due to differences in their genetic backgrounds. Similar differences have been reported previously $(8,11)$.

The glycemic profile of MGLUT4 KO mice infused with glucose into the portal vein was characterized, first, by a slightly higher glycemia in the fasted state (Figure 1a). Second, hyperglycemia developed to reach a maximum of $8.3 \pm 0.6 \mathrm{mM}$ after $60 \mathrm{~min}$ of glucose infusion and then progressively decreased, but remained higher than that of controls at the end of the experiment. The glucose AUC was negative in control and positive in the MGLUT4 KO mice.

When glucose was infused into the portal vein of the MIRKO mice, hypoglycemia developed similarly to the controls, and the corresponding glucose AUCs were negative and not different from each other (Figure 1b). When MIRKO mice and their respective controls were infused with glucose through the femoral vein, hyperglycemia transiently developed and returned to the preinfusion value after $3 \mathrm{~h}$ (data not shown), and no differences were noted between both groups of mice. This indicated that hypoglycemia induced in portal vein glucose-infused MIRKO mice was due to specific stimulation of the portal sensor.

The infusion of glucose into the portal vein of the AMPK-DN mice did not induce hypoglycemia (Figure 1c). In contrast, glycemia increased and reached a maximum $(7.4 \pm 0.1 \mathrm{mM})$ after 20 minutes, then decreased very progressively to $5.7 \pm 0.4 \mathrm{mM}$ at completion of the glucose infusion, but remained higher than in the fasted state. The AUC of the AMPK-DN mice was positive and significantly higher than that of the corresponding controls. A similar glucose infusion was also performed through the femoral vein (not shown). The blood glucose profiles were similar to each other and showed a transient hyperglycemia followed by a return of the glycemia toward the fasted levels.

Glucose clearance rates during hepatoportal glucose infusions. To evaluate if the different blood glucose profiles were associated with variations of the glucose turnover rates glucose or saline was infused into the portal vein while ${ }^{3} \mathrm{H}$-glucose was infused through the femoral vein. Measurement of isotopic dilution allowed determination of whole body glucose turnover and calculation of glucose clearance (Figure 2). The glucose clearance rates of glucose-infused control mice were similar in all groups $(79.6 \pm 11.4 ; 85.0 \pm 6.6 ; 66.4 \pm 3.9 \mathrm{ml} / \mathrm{kg} / \mathrm{min}$ in MGLUT4, MIRKO, and AMPK-DN control mice, respectively). The glucose clearance rate was reduced in the MGLUT 4 by $45 \%$ as compared with their respective controls. Glucose clearance was similar to the control value for the MIRKO mice and reduced by more than $50 \%$ in the AMPK-DN mice relative to control mice. Glucose clearance rates were higher in the glucoseinfused than in the saline-infused mice in all groups of control animals.

Plasma insulin concentrations during hepatoportal glucose infusions. Plasma insulin concentrations were assessed in all groups of mice before and 10, 30, 50, 120 , and 180 minutes after the beginning of glucose infusion into the hepatoportal vein. Plasma insulin increased in every group and remained elevated during the entire infusion period (Figure 3 and Table 1). The absolute value of plasma insulin secretion, however, was different, according to the strain of mice studied. When glucose was infused in the MGLUT4 mice, plasma insulin levels rose to reach a maximum at $50 \mathrm{~min}$ (Figure $3 \mathrm{a}$ ) and remains high until completion of the experiment. The AUC and the plasma insulin levels were significantly higher in the

Table 1

Blood glucose and plasma insulin concentrations during portal saline or glucose infusions in control and mutant mice

\begin{tabular}{|c|c|c|c|c|c|c|c|}
\hline & & \multicolumn{2}{|c|}{ MGLUT4 } & \multicolumn{2}{|c|}{ MIRKO } & \multicolumn{2}{|c|}{ AMPK-DN } \\
\hline & & T0 & T180 & T0 & T180 & T0 & T180 \\
\hline \multicolumn{8}{|c|}{ Saline infused } \\
\hline \multirow[t]{2}{*}{ Glucose (mM) } & Controls & $5.0 \pm 0.4$ & $3.8 \pm 0.8$ & $5.2 \pm 0.6$ & $4.4 \pm 0.4$ & $5.3 \pm 0.9$ & $4.1 \pm 0.6$ \\
\hline & Mutants & $6.1 \pm 0.7$ & $4.5 \pm 0.8$ & $5.8 \pm 0.8$ & $4.0 \pm 0.9$ & $5.3 \pm 0.7$ & $4.9 \pm 0.7$ \\
\hline \multirow[t]{2}{*}{ Insulin $(\mu \mathrm{U} / \mathrm{ml})$} & Controls & NA & NA & NA & NA & NA & NA \\
\hline & Mutants & NA & NA & NA & NA & NA & NA \\
\hline \multicolumn{8}{|c|}{ Glucose infused } \\
\hline \multirow[t]{2}{*}{ Glucose (mM) } & Controls & $4.8 \pm 0.7$ & $2.8 \pm 0.2$ & $5.3 \pm 0.3$ & $3.3 \pm 0.1$ & $5.3 \pm 0.1$ & $3.4 \pm 0.2$ \\
\hline & Mutants & $5.9 \pm 0.5$ & $4.0 \pm 0.5$ & $5.7 \pm 0.3$ & $3.2 \pm 0.2$ & $5.8 \pm 0.2$ & $5.7 \pm 0.4$ \\
\hline \multirow[t]{2}{*}{ Insulin $(\mu \mathrm{U} / \mathrm{ml})$} & Controls & $16.1 \pm 2.3$ & $20.5 \pm 4.2$ & $9.3 \pm 1.7$ & $19.6 \pm 1.9$ & $10.6 \pm 0.5$ & $15.1 \pm 1.3$ \\
\hline & Mutants & $18.9 \pm 1.2$ & $32.5 \pm 2.3^{\mathrm{A}}$ & $16.0 \pm 1.7$ & $22.3 \pm 9.4$ & $9.5 \pm 0.5$ & $21.1 \pm 3.7$ \\
\hline
\end{tabular}

AStatistically different from controls; $P<0.05$. 


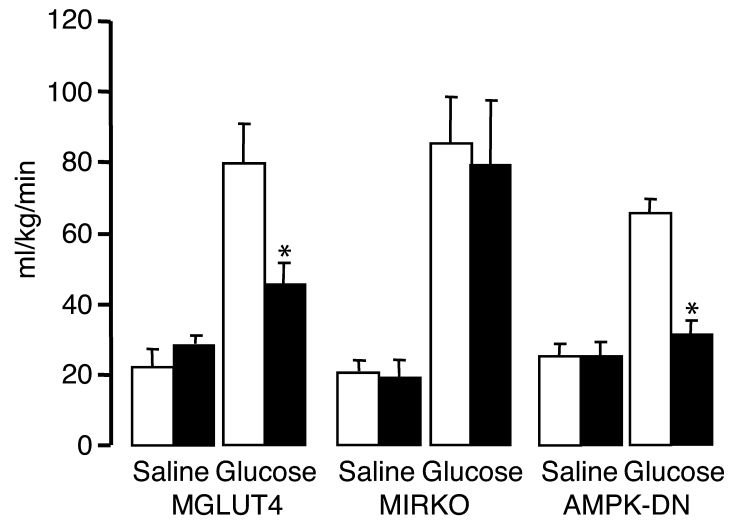

MGLUT4 than in the corresponding control mice after 30,50 , and at $180 \mathrm{~min}$. Interestingly, plasma insulin concentration was higher in the fasted state in the MIRKO as compared with control mice, but did not increase over time during the glucose infusion. Hence, the corresponding AUC, an index of the increased insulinemia during the infusion, was very small when compared with the corresponding controls (Figure $3 \mathrm{~b}$ ). The plasma insulin concentration of the AMPK-DN glucose-infused mice rose and reached values similar to the corresponding controls (Figure 3c), even though the blood glucose levels were higher in the mutant as compared with the control mice. At present, we do not have any explanation for this discrepancy between glucose and insulin levels in the mutant mice.

\section{Figure 2}

Blood glucose clearance of MGLUT4, MIRKO, and AMPK-DN mice and their respective controls infused with saline or glucose into the portal vein. Saline or glucose was infused through the portal vein in controls (white bars) and mutant mice (black bars) for 180 min at a rate of $\sim 25 \mathrm{mg} / \mathrm{kg} / \mathrm{min}$. A tritiated glucose coinfusion was performed to calculate whole body glucose turnover. Glucose clearance rates were then calculated as mean $\pm \mathrm{SE}(\mathrm{ml} / \mathrm{kg} / \mathrm{min})$. Glucose-infused mice were studied in groups of eight to ten, and saline-infused mice were studied in groups of three to four. ${ }^{*}$ Statistically different from controls; $P<0.05$.

Individual tissue glucose use. To evaluate if the reduced glucose turnover rate of the glucose-infused MGLUT4 mice was associated with a decreased muscle glucose use we injected ${ }^{14} \mathrm{C}-2 \mathrm{DG}$ in the MGLUT4 and the corresponding control mice $2 \mathrm{~h}$ after the initiation of the portal vein glucose infusion. The glucose use rates were lower in all the muscles of MGLUT4 mice as compared with the corresponding controls (Figure 4a). Conversely, the glucose use rate was normal in white and brown adipose tissues and the skin. In the MIRKO mice, no differences were noted for any tissue, except in white adipose tissue, between the control and MIRKO mice (Figure 4b). In the AMPK-DN mice, when compared with controls, the glucose use rate was reduced in most of the muscles but not in adipose tissues where it was similar (Figure 4c).
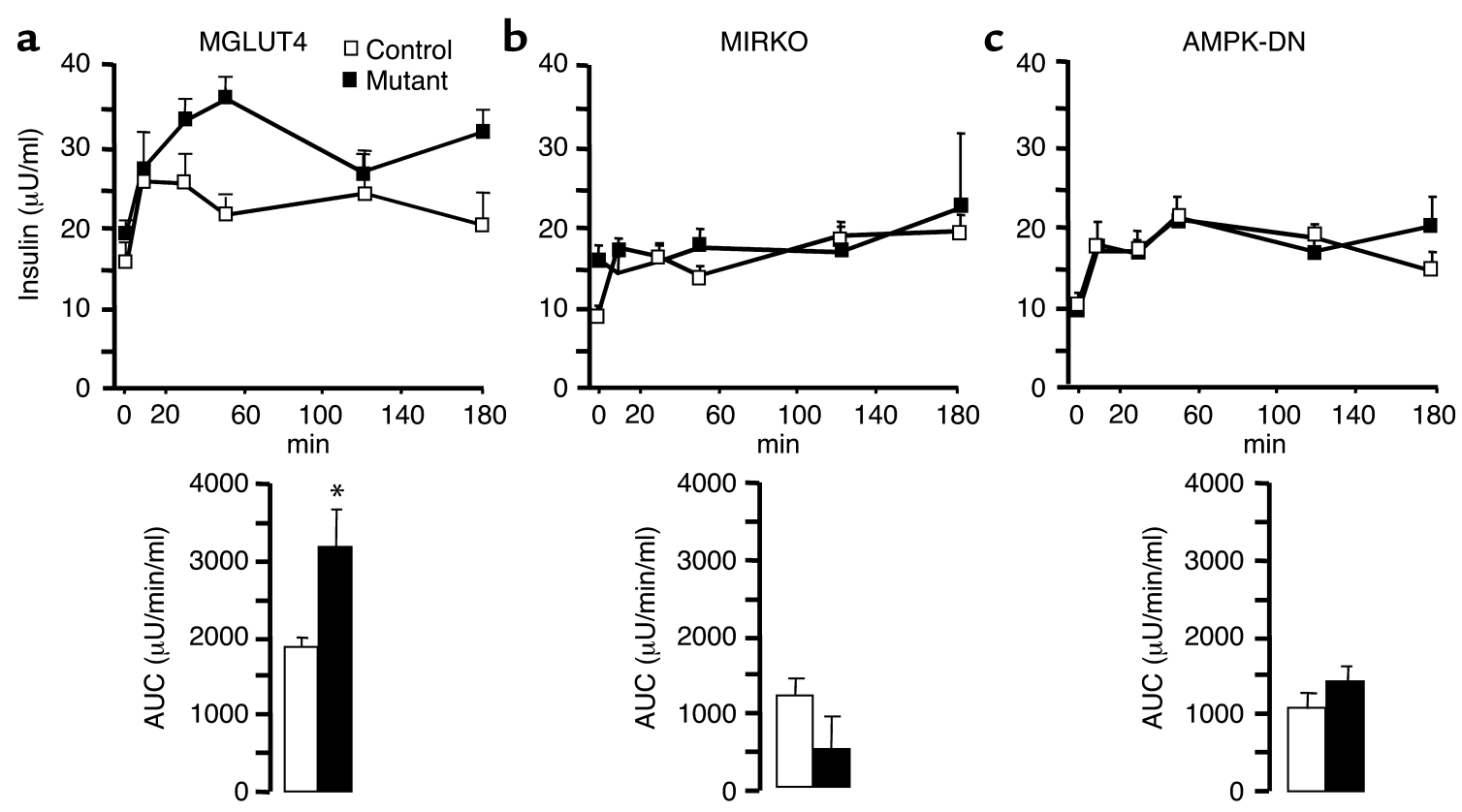

Figure 3

Plasma insulin levels of MGLUT4, MIRKO, and AMPK-DN mice and their respective controls infused with glucose into the portal vein. Glucose was infused through the portal vein in control (white bars) and mutant mice (black bars) for $180 \mathrm{~min}$ at a rate of $\sim 25 \mathrm{mg} / \mathrm{kg} / \mathrm{min}$. Plasma insulin levels were determined from the tip of the tail vein before and 10, 30, 50,120, and 180 min after the beginning of the glucose infusion and are reported as mean $\pm \mathrm{SE}$ for eight to ten mice per group. The insets depict the corresponding AUC ( $\mu U / \mathrm{min} / \mathrm{ml})$ for the different groups of mice. (a) MGLUT4 mice; (b) MIRKO mice; (c) AMPK-DN mice. ${ }^{*}$ Statistically different from controls; $P<0.05$. 


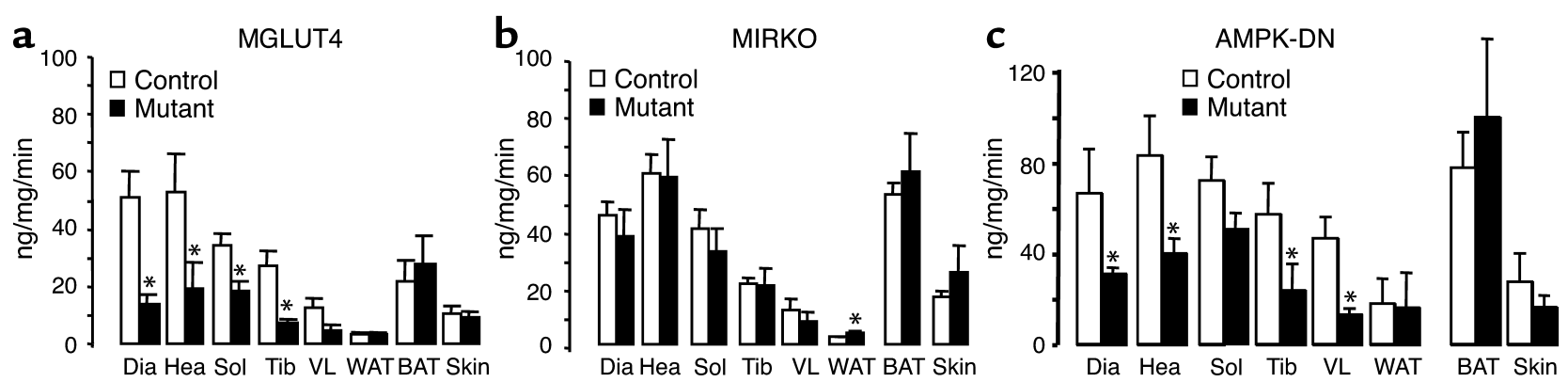

\section{Figure 4}

Glucose use rates of MGLUT4, MIRKO, and AMPK-DN mice and their respective controls infused with glucose into the portal vein. Glucose was infused through the portal vein in control (white bars) and mutant mice (black bars) for $180 \mathrm{~min}$ at a rate of $\sim 25 \mathrm{mg} / \mathrm{kg} / \mathrm{min}$. A ${ }^{14} \mathrm{C}-2 \mathrm{DG}$ injection protocol was used to determine glucose use $(\mathrm{ng} / \mathrm{mg} / \mathrm{min}$ ) by the diaphragm (Dia), the heart (Hea), the soleus muscle (Sol), the tibialis anterior (Tib) the vastus lateralis (VL), the white gonadal tissue (WAT), the brown interscapular adipose tissue (BAT), and the skin. (a) MGLUT4 mice; (b) MIRKO mice; (c) AMPK-DN mice. Eight to ten mice per group were studied. *Statistically different from controls; $P<0.05$.

\section{Discussion}

Glucose infusion into the hepatoportal vein stimulates glucose use in muscle and brown adipose tissue and leads to development of hypoglycemia. In the present study, using genetically modified mice we show that the signal transmitted by the hepatoportal sensor depends on muscle expression of GLUT4 and the AMPK, but not on the presence of an insulin receptor.

The role of GLUT4 in hepatoportal sensor-stimulated muscle glucose use was evaluated in mice with muscle-specific inactivation of GLUT4. Infusion of glucose into the portal vein of these mice induced a glycemic profile characterized by a transient rise in blood glucose concentration, which was very similar to that observed when glucose was infused in the femoral vein, i.e., without activation of the hepatoportal sensor (8). In addition, the glucose clearance rate was lower than for the corresponding controls. It was, nevertheless, higher than in the saline-infused mice. Individual tissue glucose use was reduced in all muscles, but not in the adipose tissues. Taken together, these data show that maximal activation by the hepatoportal glucose sensor of muscle glucose use requires the presence of GLUT4 in this tissue. Nevertheless, since glucose clearance was not reduced to the level of saline-infused mice, whereas it was reduced to this level in AMPK mutant mice, this suggests that other glucose transporters could participate in muscle glucose use in response to the activation of the hepatoportal sensor. The identity of these transporters is not known but they may belong to the family of recently cloned transporter isoforms $(23,24)$.

Another possible explanation for the decreased portal sensor-stimulated clearance in MGLUT4 mice could be related to the reported glucotoxicityinduced insulin resistance that develops on these mice (25). In this cited study, however, this secondary complication was seen only in aged male mice. Here, we used younger female mice, which had homogenous, nondiabetic, blood glucose levels and which did not display glutotoxicity-induced insulin resistance, as confirmed by normal rates of ${ }^{14} \mathrm{C}-2 \mathrm{DG}$ uptake in white and brown adipose tissue during the portal vein glucose infusion (Figure 4a). Thus, impairment of glucose clearance secondary to glucotoxicity is unlikely to explain any of the modifications in the parameters we measured.

In MIRKO mice, hypoglycemia developed as in the corresponding control animals. In addition, glucose clearance was increased to the same extent in both groups of mice. These data strongly suggest that muscle glucose use activated by the portal vein sensor is insulin-independent. This observation may also explain the previous report that MIRKO mice have a normal tolerance to an intraperitoneal glucose administration (26). In this particular situation, glucose is rapidly collected into the portal vein where it stimulates the glucose sensor and, consequently, induces glucose uptake in muscle. Earlier data showing that intraperitoneal glucose injection-induced transient hypoglycemia in the rat (5) are compatible with this interpretation.

The insulin-independent increase in GLUT4dependent muscle glucose use indicates that an alternative intracellular signaling pathway must be activated to stimulate GLUT4 translocation to the plasma membrane. AMPK appears to play an essential role in stimulating GLUT4 translocation during contraction or hypoxia $(16,20,27)$. To determine if AMPK was recruited following activation of the hepatoportal sensor, we used mice expressing a dominant negative AMPK transgene in muscle (20). Glucose infusion into the portal vein of these mice led to development of hyperglycemia, which persisted throughout the glucose infusion. In addition, the glucose clearance rate was reduced almost to the level of the saline-infused mice. Individual muscle glucose use was also reduced in muscles of the AMPK-DN mice. These data thus indicate that muscle AMPK is a key signaling intermediate in the transmission of the signal generated at the portal sensor. This is also supported by the observation that the activity of the 
a2-AMP-activated kinase was increased $20 \%$ at the end of the 3-h infusion period in the heart and muscles of the portal glucose-infused mice, although the difference did not reach statistical significance (not shown). Further analysis of AMPK activation by Western blot analysis of its phosphorylated form performed at 20,60, and $180 \mathrm{~min}$ after initiation of portal vein glucose infusion did not show statistically significant increased phosphorylation. This is, however, not too surprising when considering that the kinetics of hypoglycemia induction is slow and much slower than that induced by AICAR administration. Also, there was a basal level of AMPK phosphorylation in saline-infused mouse muscle. This could be due to the fact that the mice were starved for $6 \mathrm{~h}$ before the beginning of the experiment and probably also to the stress and hypoxia induced by the surgical and muscle isolation procedures. Thus, a small activation of AMP kinase by the activated portal sensor may be masked. Nevertheless, our data indicate that activity of the AMP kinase is required in muscle for portal vein sensor-stimulated glucose uptake.

Recent studies described activation of muscle AMPK by an intracerebroventricular injection of leptin $(28,29)$. In addition, we and others, have shown that leptin stimulates muscle glucose use (30-32). These mechanisms involved the activation of the autonomic nervous system, as does the activation of the hepatoportal sensor transmitting the glucose signal toward the target tissues. These data reinforce our hypothesis that the AMP-activated kinase is involved in the activation of muscle glucose use by the activated hepatoportal glucose sensor.

In conclusion, the present study extends our understanding of the regulation of glucose use in muscles by the hepatoportal glucose sensor. First, our previous studies using mutant mice demonstrated that this sensor was GLUT2 dependent and required the presence of an activated GLP-1 receptor for full glucose competence. This sensor was also inhibited by somatostatin (8). Electrophysiological studies have demonstrated that activation of the portal sensor by glucose leads to changes in the firing rate of hepatic afferent branches of the vagus nerve $(33,34)$. These project to the nucleus of the tractus solitarius and the ventromedial and lateral hypothalamus $(28,35$, 36 ). There is thus a direct connection between the portal sensor and central brain areas involved in the control of the autonomic nervous system regulating food intake, glucose uptake by peripheral tissues, and glucagon secretion. Here, we further demonstrate, using genetically-modified mice, that the signal transmitted at the muscle level does not require the presence of an insulin receptor, but requires the presence of GLUT4. In addition, activation of glucose transport is suppressed by expression of the AMPK-DN in muscle, indicating a requirement for AMP kinase activity in the stimulation of glucose uptake. This description of a glucose sensor, able to induce hypoglycemia in an insulin-independent fashion, demonstrates an additional complexity in the control of glucose homeostasis. This sensor may play a particularly important role in the postprandial state when a portal-peripheral glucose gradient is established. Dysfunction of this sensor may cause impaired glucose tolerance in the prediabetic or diabetic states. Its function may also become progressively blunted in diabetes, when peripheral hyperglycemia is less well controlled and a portalperipheral gradient more difficult to establish.

\section{Acknowledgments}

This work was supported by grants from the Swiss National Science Foundation to R. Burcelin and B. Thorens, from the Juvenile Diabetes Research Foundation and the Association de Langue Française pour l'Etude du Diabète et des Maladies Métaboliques (sponsored by SmithKline Beecham) to R. Burcelin, and NIH grant DK-4305 to B.B. Kahn. We would like to thank F. Mauvais-Jarvis, L. Pénicaud, and L. Casteilla for helpful criticisms, and Barbara Menard and Odile Peroni for their technical expertise.

1. Moore, M.C., and Cherrington, A.D. 1996. The nerves, the liver, and the route of feeding: an integrated response to nutrient delivery. Nutrition. 12:282-284.

2. Jungermann, K., et al. 1986. Regulation of liver metabolism by the hepatic nerves. In Advances in enzymes regulation, G. Weber, editor. Pergamon Press. Oxford, United Kingdom. 63-88.

3. Stümpel, F., and Jungermann, K. 1997. Sensing by intrahepatic muscarinic nerves of a portal-arterial glucose concentration gradient as a signal for insulin-dependent glucose uptake in the perfused rat liver. FEBS. Lett. 406:119-122.

4. Russek, M. 1963. Participation of hepatic glucoreceptors in the control of intake of food. Nature. 197:79-80.

5. Russek, M. 1970. Demonstration of the influence of an hepatic glucosensitive mechanism on food-intake. Physiol. Behav. 5:1207-1209.

6. Hamilton-Wessler, M., Bergman, R.N., Halter, J.B., Watanabe, R.M., and Donovan, C.M. 1994. The role of liver glucosensors in the integrated sympathetic response induced by deep hypoglycemia in dogs. Diabetes. 43:1052-1060.

7. Donovan, C.M., Hamilton-Wessler, M., Halter, J.B., and Bergman, R.N. 1994. Primacy of liver glucosensors in the sympathetic response to progressive hypoglycemia. Proc. Natl. Acad. Sci. U. S. A. 91:2863-2867.

8. Burcelin, R., Dolci, W., and Thorens, B. 2000. Portal glucose infusion in the mouse induces hypoglycemia. Evidence that the hepatoportal glucose sensor stimulates glucose utilization. Diabetes. 49:1635-1642.

9. Burcelin, R., Dolci, W., and Thorens, B. 2000. Glucose sensing by the hepatoportal sensor is GLUT2-dependent. In vivo analysis in GLUT2-null mice. Diabetes. 49:1643-1648.

10. Thorens, B., Guillam, M.-T., Beermann, F., Burcelin, R., and Jaquet, M. 2000. Transgenic reexpression of GLUT1 or GLUT2 in pancreatic $\beta$ cells rescues Glut 2 -null mice from early death and restores normal glucose-stimulated insulin secretion. J. Biol. Chem. 275:23751-23758.

11. Burcelin, R., DaCosta, A., Drucker, D., and Thorens, B. 2001. Glucose competence of the hepatoportal vein sensor requires the presence of an activated GLP-1 receptor. Diabetes. 50:1720-1728.

12. Rea, S., and James, D.E. 1997. Moving GLUT4-The biogenesis and trafficking of GLUT4 storage vesicles. Diabetes. 46:1667-1677.

13. Kahn, B.B. 1996. Lilly lecture 1995. Glucose transport: pivotal step in insulin action. Diabetes. 45:1644-1654.

14. Sherman, L.A., Hirshman, M.F., Cormont, M., Le Marchand-Brustel, Y., and Goodyear, L.J. 1996. Differential effects of insulin and exercise on Rab4 distribution in rat skeletal muscle. Endocrinology. 137:266-273.

15. Goodyear, L.J., and Kahn, B.B. 1998. Exercise, glucose transport, and insulin action. Annu. Rev. Med. 49:235-261.

16. Merrill, G.F., Kurth, E.J., Hardie, D.G., and Winder, W.W. 1997. AICA riboside increases AMP-activated protein kinase, fatty acid oxidation, and glucose uptake in rat muscle. Am. J. Physiol. 273:E1107-E1112. 
17. Kurth-Kraczek, E.J., Hirshman, M.F., Goodyear, L.J., and Winder, W.W. 1999. 5'-AMP-activated protein kinase activation causes GLUT4 translocation in skeletal muscle. Diabetes. 48:1667-1671.

18. Hayashi, T., et al. 2000. Metabolic stress and altered glucose transport: activation of AMP-activated protein kinase as a unifying coupling mechanism. Diabetes. 49:527-531.

19. Fujii, N., et al. 2000. Exercise inducesisoform-specific increase in 5 'AMP-activated protein kinase in human skeletal muscle. Biochem. Biophys. Res. Comm. 273:1150-1155.

20. Mu, J., Brozinik, J.T., Jr., Valladares, O., Bucan, M., and Birnbaum, M.J. 2001. A role of AMP-activated protein kinase in contraction- and hypoxia-regulated glucose transport in skeletal muscle. Mol. Cell. 7:1085-1094.

21. Stenbit, A.E., et al. 1997. Diverse effects of GLUT4 ablation on glucose uptake and glycogen synthesis in red and white skeletal muscle. J. Clin. Invest. 98:629-634.

22. Zisman, A., et al. 2000. Targeted disruption of the glucose transporter 4 selectively in muscle causes insulin resistance and glucose intolerance. Nat. Med. 6:924-928.

23. Ibberson, M., Uldry, M., and Thorens, B. 2000. GLUTX1, a nove mammalian glucose transporter expressed in the central nervous system and insulin-sensitive tissues. J. Biol. Chem. 275:4607-4612.

24. Joost, H.G., and Thorens, B. 2001. The extended GLUT-family of sugar/polyol transport facilitators: nomenclature, sequence characteristics, and potential function of its novel members. Mol. Membr. Biol. 18:247-256.

25. Kim, J.K., et al. 2001. Glucose toxicity and the development of diabetes in mice with muscle-specific inactivation of GLUT4. J. Clin. Invest. 108:153-160. doi:10.1172/JCI200110294.

26. Brüning, J.C., et al. 1998. A muscle-specific insulin receptor knockout exhibits features of the metabolic syndrome of NIDDM without altering glucose tolerance. Mol. Cell. 2:559-569.

27. Hardie, D.G., Salt, I.P., Hawley, S.A., and Davies, S.P. 1999. AMP-activated protein kinase: an ultrasensitive system for monitoring cellular energy charge. Biochem. J. 338:717-722.

28. Minokoshi, Y., et al. 2002. Leptin stimulates fatty-acid oxidation by activating AMP-activated protein kinase. Nature. 415:339-343.

29. Kim, Y.B., Uotani, S., Pierroz, D.D., Flier, J.S., and Kahn, B.B. 2000. In vivo administration of leptin activates signal transduction directly in insulin-sensitive tissues: overlapping but distinct pathways from insulin. Endocrinology. 141:2328-2339.

30. Kamohara, R., Burcelin, R., Halaas, J.L., Friedman, J.M., and Charron, M.J. 1997. Acute stimulation of glucose metabolism in mice by leptin treatment. Nature. 389:374-377.

31. Minokoshi, Y., Okano, Y., and Shimazu, T. 1994. Regulatory mechanism of the ventromedial hypothalamus in enhancing glucose uptake in skeletal muscle. Brain Res. 649:343-347.

32. Minokoshi, Y., Haque, M.S., and Shimazu, T. 1999. Microinjection of leptin into the ventromedial hypothalamus increases glucose uptake in peripheral tissues in rats. Diabetes. 48:287-291.

33. Niijima, A. 1969. Afferent impulse discharges from glucoreceptors in the liver of the guinea pig. Ann. N. Y. Acad. Sci. 157:690-700.

34. Niijima, A. 1984. The effect of D-glucose on the firing rate of glucose-sensitive vagal afferents in the liver in comparison with the effect of 2-deoxy-D-glucose. J. Auton. Nerv. Syst. 10:279-285.

35. Shimizu, N., Oomura, Y., Novin, D., Grijalva, C.V., and Cooper, P.H. 1983. Functional correlations between lateral hypothalamic glucose-sensitive neurons and hepatic portal glucose-sensitive units in rat. Brain Res. 265:49-54.

36. Schmitt, M. 1973. Influences of hepatic portal receptors on hypothalamic feeding and satiety centers. Am. J. Physiol. 225:1089-1095 